\title{
Microstructure and charge carrier transport in phthalocyanine based semiconductor blends
}

\author{
Andreas Opitz ${ }^{1}$, Julia Wagner ${ }^{1}$, Bernhard Ecker ${ }^{1}$, Ulrich Hörmann ${ }^{1}$, Michael Kraus ${ }^{1}$, Markus \\ Bronner $^{1}$, Wolfgang Brütting ${ }^{1}$, Alexander Hinderhofer ${ }^{2}$, Frank Schreiber ${ }^{2}$ \\ ${ }^{1}$ Institute of Physics, University of Augsburg, Augsburg, Germany. \\ ${ }^{2}$ Institute of Applied Physics, University of Tübingen, Tübingen, Germany.
}

\begin{abstract}
The continuously growing and wide-spread utilization of blends of organic electron and hole conducting materials comprises ambipolar field-effect transistors as well as organic photovoltaic cells. Structural, optical and electrical properties are investigated in blends and neat films of the electron donor material $\mathrm{Cu}$-phthalocyanine $(\mathrm{CuPc})$ together with fullerene $\mathrm{C}_{60}$ and $\mathrm{Cu}$-hexadecafluorophthalocyanine $\left(\mathrm{F}_{16} \mathrm{CuPc}\right)$ as electron acceptor materials, respectively. The difference in molecular structure of the spherical $\mathrm{C}_{60}$ and the planar molecule CuPc leads to nanophase separation in the blend, causing charge carrier transport which is limited by the successful formation of percolation paths. In contrast, blends of the similar shaped CuPc and $\mathrm{F}_{16} \mathrm{CuPc}$ molecules entail mixed crystals, as can be clearly seen by X-ray diffraction measurements. We discuss differences of both systems with respect to their microstructure as well as their electrical transport properties.
\end{abstract}

\section{INTRODUCTION}

From the mid 1990s the concept of "bulk-heterojunction solar cells" revolutionized the field of organic photovoltaics: Yu et al. reported a polymeric solar cell with an interpenetrating donor/acceptor material system which enables a spatially distributed interface accounting for the small exciton diffusion lengths in organic semiconducting materials [1]. Since that time, blends of organic electron and hole conductive materials are widely used for ambipolar charge carrier transport and photovoltaic cells. The application of distributed interfaces in organic solar cells has the advantage that excitons can efficiently dissociate throughout the whole volume of the organic layer yielding higher amounts of free charge carriers as compared to a bilayer system. Nevertheless, for an efficient transport, each material must provide continuous paths to the contacts. Both aspects entail a competition between efficient charge carrier dissociation and preferably undisturbed transport properties inside the blend.

In this study we present the analysis of two model systems for donor-acceptor blends. These are (i) $\mathrm{Cu}$-phthalocyanine $(\mathrm{CuPc})$ combined with the Buckminster fullerene $\mathrm{C}_{60}$ and (ii) $\mathrm{CuPc}$ in combination with its fluorinated counterpart $\mathrm{F}_{16} \mathrm{CuPc}$. While $\mathrm{CuPc}$ acts as the donor or p-conductor, $\mathrm{C}_{60}$ and $\mathrm{F}_{16} \mathrm{CuPc}$ are the n-conducting acceptor materials. In addition to studying the fundamental structural and optical properties, centering on the question of phase separation or formation of mixed crystals, we extend our analysis to electrical charge carrier transport properties. The materials used have been previously investigated in similar configurations partially with different donor or acceptor materials [2,3], however, no systematic comparison with respect to the influence of the mixing behavior on the transport properties was reported. 


\section{EXPERIMENT}

Diodes for ambipolar charge carrier transport and unipolar hole-only devices were fabricated on commercially available indium-tin-oxide (ITO)-coated glass substrates (Merck) which were cleaned with different solvents in an ultrasonic bath followed by an oxygen plasma treatment in order to enhance the work function of ITO and to improve wetability for the aqueous suspension of the intrinsically conducting polymer poly(3,4-ethylenedioxythiophene): poly(styrenesulfonate) (PEDOT:PSS, purchased from H.C. Starck as BAYTRON P). The organic layers were grown by vacuum deposition from low-temperature effusion cells with a base pressure of about $10^{-7}$ mbar and deposition rates between $0.35 \AA / s$ for neat films and up to $1.4 \AA / \mathrm{s}$ for the material with the higher volume fraction in the mixtures.

For the ambipolar setup, a thin ( $5 \AA$ ) layer of LiF was deposited prior to deposition of the Al cathode which was evaporated through a shadow mask to a thickness of $1000 \AA$, giving an active area of $2 \times 2 \mathrm{~mm}^{2}$. For hole-only devices, the organic semiconductor is sandwiched between a PEDOT:PSS coated ITO substrate and a Au counterelectrode combined with an electron blocking layer consisting of a $40 \mathrm{~nm}$ thick layer of N,N'-bis(3-methylphenyl)-(1,1'biphenyl)-4,4'-diamine (TPD) or alternatively a thin layer of 2,3,5,6-tetrafluoro-7,7,8,8tetracyano-quinodimethane $\left(\mathrm{F}_{4} \mathrm{TCNQ}\right)$. For pure electron transport the organic layer is framed between an $\mathrm{Al}$ electrode at the bottom and a $\mathrm{LiF} / \mathrm{Al}$ counterelectrode on top, restricting the injection to electrons. The overall thickness of the organic layer amounts to $200 \mathrm{~nm}$ for the $\mathrm{CuPc} / \mathrm{C}_{60}$ system and $80 \mathrm{~nm}$ for the $\mathrm{CuPc} / \mathrm{F}_{16} \mathrm{CuPc}$ system. The structural formulas of the materials used are depicted in figure 1.

$\mathrm{CuPc}$ and $\mathrm{F}_{16} \mathrm{CuPc}$ were purchased from Sigma Aldrich as sublimed grade and additionally purified by thermal gradient sublimation prior to deposition. $\mathrm{C}_{60}$ was purchased from Sigma Aldrich as sublimed grade and used as received. The mixed layers were grown by codeposition from independent evaporation sources, with the deposition rates monitored by two quartz-crystal microbalances.

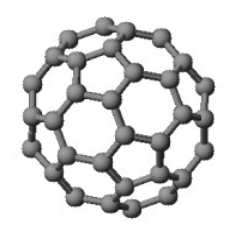

(a)

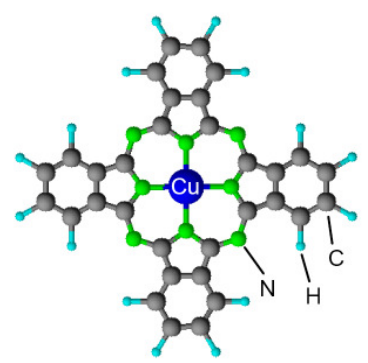

(b) (c)

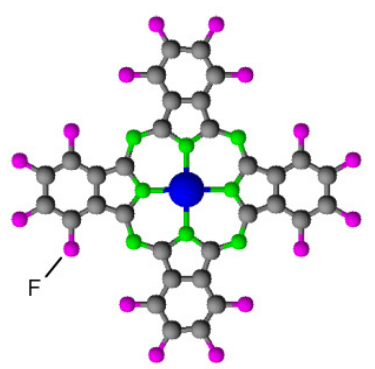

Figure 1. Chemical structure of (a) buckminsterfullerene $\mathrm{C}_{60}$, (b) Cu-phthalocyanine (CuPc) and (c) fluorinated $\mathrm{Cu}$-phthalocyanine $\left(\mathrm{F}_{16} \mathrm{CuPc}\right)$.

Assuming insulating organic films without intrinsic charge carriers and traps, the quantitative analysis of the current-voltage characteristics was realized using the model of trapfree space charge limited currents [4] extended by the Pool-Frenkel like field-dependence of the mobility [5], resulting in a current density given by

$$
j_{S C L C}=\frac{9}{8} \varepsilon \mu_{0} \frac{V_{e f f}^{2}}{d^{3}} \exp \left(0.89 \beta \sqrt{\frac{V_{e f f}}{d}}\right) \text {, }
$$


with the zero-field mobility $\mu_{0}$, the field activation parameter $\beta$ and the layer thickness $d$. In order to account for a built-in potential $V_{\mathrm{bi}}$ caused by electrodes with different work-functions, an effective voltage $V_{\text {eff }}$ is considered which equals the externally applied voltage reduced by $V_{\text {bi }}$. The parameters $\mu_{0}, V_{\mathrm{bi}}$ and $\beta$ are determined by fitting the measured current-voltage characteristics in the higher voltage range.

Optical absorption spectra were measured on neat and blended films deposited on ITOcoated glass substrates covered with PEDOT:PSS using a Varian Cary 50 UV/Visspectrophotometer. The X-ray scattering measurements were conducted on a GE/Seifert X-ray diffractometer $(\mathrm{Cu} \mathrm{K \alpha} 1$ radiation, multilayer mirror, and double bounce compressor monochromator). While electrical characterization could be realized without exposure to air, X-ray scattering as well as absorption measurements have been performed under ambient atmosphere.

\section{RESULTS and DISCUSSION}

Structural properties $\mathrm{X}$-ray scattering measurements, performed in $\theta-2 \theta$ geometry, for neat, mixed and bilayered films are depicted in figure 2 for the material combination $\mathrm{CuPc} / \mathrm{C}_{60}$ $(\mathrm{a}, \mathrm{b})$ and for $\mathrm{CuPc} / \mathrm{F}_{16} \mathrm{CuPc}(\mathrm{c})$. Both types of phthalocyanines show well pronounced diffraction peaks. In addition to previous measurements [6,7], the parallelized and monochromated incident beam enables the detection of the $\mathrm{C}_{60}$ diffraction peak.

The diffraction pattern of the $\mathrm{CuPc} / \mathrm{C}_{60}$ blend displays the same peak positions as detected for the respective neat films and was in the literature assigned to the (200)-reflection of the $\alpha$-phase of CuPc [8] and the (111)-peak of the fcc structure of $\mathrm{C}_{60}$. This observation rules out the formation of a solid solution but is a clear indicator of phase separation with coexisting crystallites of both materials. This can be ascribed to the different molecular structures of the flat $\mathrm{CuPc}$ molecule and the spherical $\mathrm{C}_{60}$. By contrast, the similar molecular structure of $\mathrm{CuPc}$ and $\mathrm{F}_{16} \mathrm{CuPc}$ leads to the formation of a mixed crystal in the blend clearly visible by a diffraction peak which is positioned between the Bragg-reflections of the neat films (see figure 2(c)). This mixed crystal may exhibit a similar structure like the neat phthalocyanines $[9,10]$.

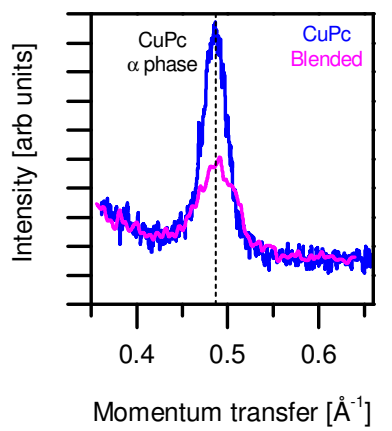

(a) Figure 2. X-ray diffraction spectra for ne
$(\mathrm{a}, \mathrm{b}) \mathrm{CuPc} / \mathrm{C}_{60}$ and the $(\mathrm{c}) \mathrm{CuPc} / \mathrm{F}_{16} \mathrm{CuPc}$.

The structural size of the crystallites can be estimated from the width of the diffraction peaks, which is of particular interest in case of phase separated crystals appearing in the $\mathrm{CuPc} / \mathrm{C}_{60}$ blend. Using the relation $\Delta q_{\mathrm{z}} \sim 2 \pi / L$ with $\Delta q_{\mathrm{z}}$ being the width of the diffraction peak, the coherence volume $L$ corresponding to the size of the crystallites can be evaluated to about 
$10 \mathrm{~nm}$ for both $\mathrm{CuPc}$ and $\mathrm{C}_{60}$. Thus, phase separation in this material system can be ascribed to a relatively small length scale in comparison to the large scale phase separation detected in the system pentacene/fullerene [11].

The film morphology of 1:1 blended films of both material combinations are depicted in figure 3, as investigated by non-contact scanning force microscopy (SFM). The materials have been deposited onto $\mathrm{SiO}_{2} / \mathrm{Si}$ or PEDOT:PSS/ITO/glass at a substrate temperature of $100{ }^{\circ} \mathrm{C}$. Even at a nominal thickness of $25 \mathrm{~nm}$, maximum heights of up to $58 \mathrm{~nm}$ are observed in the blend of $\mathrm{C}_{60}$ and $\mathrm{CuPc}$, which approves the model of demixing and phase separation proposed by $\mathrm{X}$-ray scattering. By contrast, the blended $\mathrm{CuPc} / \mathrm{F}_{16} \mathrm{CuPc}$ films show a needle-like structure with similar morphologies as the neat films [7]. This observation confirms the structural result of mixed crystals.

(a)

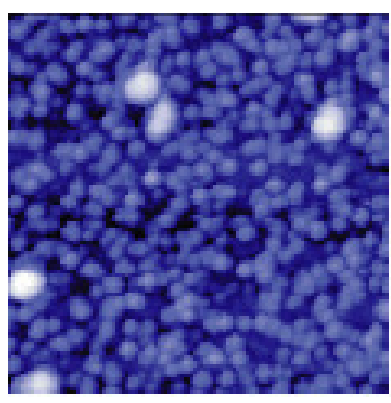

(b)

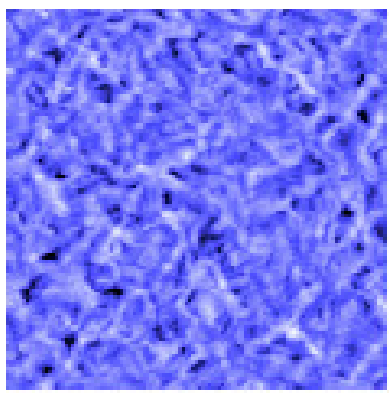

Figure 3. Scanning force microscopy images taken in non-contact mode of 1:1 blended films (nominal thickness of $25 \mathrm{~nm}$ ) of (a) $\mathrm{C}_{60}$ and $\mathrm{CuPc}$ deposited on $\mathrm{SiO}_{2} / \mathrm{Si}$ substrate (Max. height: $58 \mathrm{~nm}$, RMS roughness: $6.5 \mathrm{~nm}$ ) (b) $\mathrm{CuPc}$ and $\mathrm{F}_{16} \mathrm{CuPc}$ deposited on a PEDOT:PSS coated ITO substrate (Max. height: $15 \mathrm{~nm}$, RMS roughness: $2.0 \mathrm{~nm}$ ) with a total image size of $2 \times 2 \mu \mathrm{m}^{2}$.

Optical absorption spectra Regarding the suitability of the blends for solar cell applications, we analyzed the absorption spectra of neat films as well as blends with a mixing ratio of 1:1 of both material systems, depicted in figure 4 . Neat films of $\mathrm{CuPc}$ and $\mathrm{C}_{60}$ show complementary absorption behavior in the visible spectral range, which qualifies this material combination for application over the whole visible spectrum including the near IR. The spectrum of the blend displays a shape corresponding to a linear combination of both neat spectra, which confirms the model of phase separation [12].

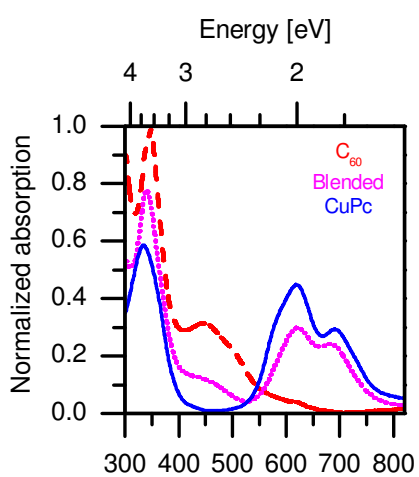
(a) Wave length [nm]

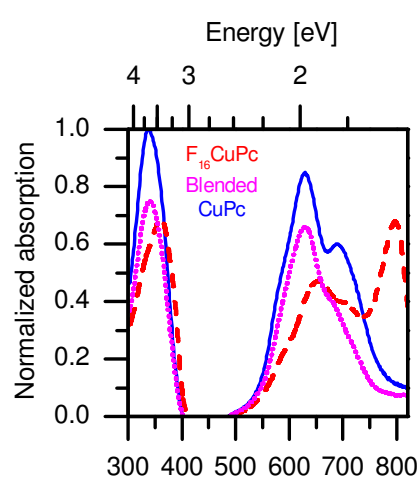

(b)

Figure 4. Absorption spectra in the UV-Vis range of neat films and $1: 1$ blends: (a) $\mathrm{CuPc} / \mathrm{C}_{60}$; (b) $\mathrm{CuPc} / \mathrm{F}_{16} \mathrm{CuPc}$. 
In contrast, both $\mathrm{CuPc}$ and $\mathrm{F}_{16} \mathrm{CuPc}$ show hardly any absorption in the blue-green wavelength range of the visible spectrum between the two absorption edges at $\lambda=400 \mathrm{~nm}$ and $550 \mathrm{~nm}$, making this material combination not very favorable for sunlight application. The slightly differing curves in the higher wavelength range can be assigned to the difference in the interaction of the molecules in the unit cell $[13,3]$. When mixing both molecules the intensity of the peak at $793 \mathrm{~nm}$, which may be attributed to a non-herringbone structure [10], is reduced drastically.

Electrical properties Investigations of electrical transport properties of neat and blended films have been realized by fabricating hole-only, electron-only and ambipolar diodes [14]. The corresponding $I-V$ characteristics are analyzed using a fit based on the trap-free SCLC model described above. The zero-field mobilities of the $\mathrm{CuPc} / \mathrm{C}_{60}$ material system in dependence on the concentration are extracted from the fit results and summarized in figure 5(a). It can be seen that both charge carrier types are transported in $\mathrm{CuPc}$ as well as in $\mathrm{C}_{60}$, even though the unipolar mobilities depend strongly on the mixing ratio. Starting from neat $\mathrm{C}_{60}$ the electron mobility decreases exponentially with increasing $\mathrm{CuPc}$ addition and shows a further reduction when switching over to neat $\mathrm{CuPc}$. These aspects allow for the conclusion that the electron transport in the blend is predominantly carried by $\mathrm{C}_{60}$ molecules and decreases in the blend where the hopping distance between the molecules is increased. The unipolar hole mobility changes uniformly over the whole concentration range. As a consequence of the ambipolar nature of both $\mathrm{CuPc}$ and $\mathrm{C}_{60}$, the ambipolar mobility in the blends is higher than the sum of unipolar hole and electron mobility. A consolidated view of these factors indicates that the charge carrier transport in blended films is mainly based on the excellent electron transport properties inside conductive paths of $\mathrm{C}_{60}$.

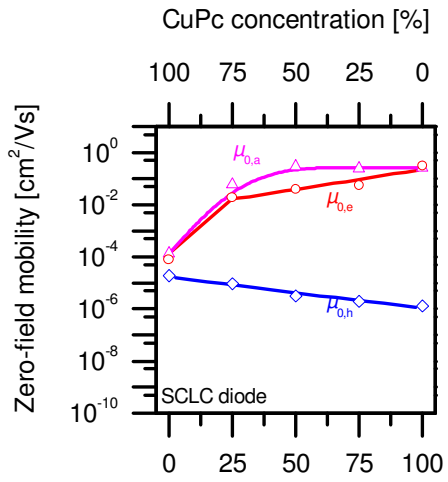

(a)

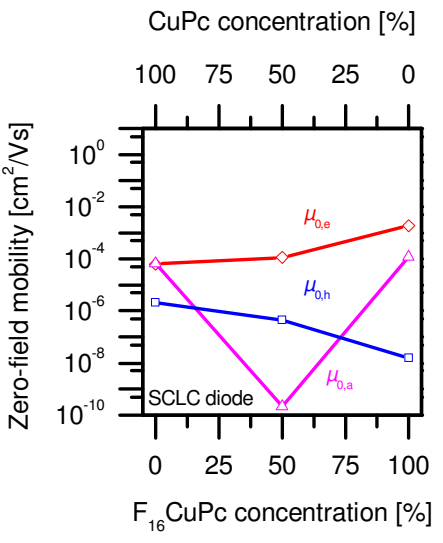

(b)

Figure 5. Zero-field mobilities for electron-only, hole-only, and ambipolar transport determined from the SCLC model for neat and blended films of $\mathrm{CuPc} / \mathrm{C}_{60}$ (a) and $\mathrm{CuPc} / \mathrm{F}_{16} \mathrm{CuPc}$ (b).

The zero-field mobilities of the $\mathrm{CuPc} / \mathrm{F}_{16} \mathrm{CuPc}$ material system are depicted in figure 5(b). The unipolar mobilities in the blended films are in-between the ones of the neat films, indicating that the blend contains mixed crystals with similar $\pi-\pi$ overlaps as compared to the neat films. In contrast to the $\mathrm{CuPc} / \mathrm{C}_{60}$ material system, the ambipolar mobility inside the $\mathrm{CuPc} / \mathrm{F}_{16} \mathrm{CuPc}$ blend is several orders of magnitude lower than both unipolar mobilities as well as the ambipolar mobilities of the neat films. Since the unipolar mobilities are high, it is hardly 
probable that the extremely low ambipolar transport in the blend is due to an absence of percolation paths. Instead, the reduced ambipolar mobility is probably related to the simultaneous presence of both charge carrier types. A tentative explanation may be found in the generation of charge transfer (CT) excitons which might be created by the injection of both charge carrier types [15]. As a result, these CT excitons would limit the transport by blocking the occupied molecules for further injected charge carriers. The generation of CT excitons can be facilitated by the high electron affinity of the $\mathrm{F}_{16} \mathrm{CuPc}$ which is located close to the ionization potential of $\mathrm{CuPc}[16]$. For proving this phenomenon further work is in progress.

\section{SUMMARY}

It has been demonstrated that blends of $\mathrm{CuPc}$ and $\mathrm{C}_{60}$ as compared to mixed films of $\mathrm{CuPc}$ and $\mathrm{F}_{16} \mathrm{CuPc}$ display different types of film growth, namely phase separation and mixed crystal formation. The transport properties of the phase separated blend of $\mathrm{CuPc} / \mathrm{C}_{60}$ are based on percolation paths of the different phases and dominated by the electron transport in $\mathrm{C}_{60}$. $\mathrm{By}$ contrast, blends of the phthalocyanines $\mathrm{CuPc}$ and $\mathrm{F}_{16} \mathrm{CuPc}$ demonstrate the formation of mixed crystals. A drastically reduced ambipolar mobility was found in the blend and might be assigned to the generation of charge transfer excitons in neighboring $\mathrm{CuPc}$ and $\mathrm{F}_{16} \mathrm{CuPc}$ molecules created by the simultaneous injection or photogeneration of both charge carrier types.

\section{ACKNOWLEDGMENTS}

This work was supported by the Deutsche Forschungsgemeinschaft through priority programs 1121 and 1355. The authors thank Jens Pflaum (Universities of Stuttgart and Würzburg) for purifying organic materials.

\section{REFERENCES}

1. G. Yu et al., Science 270, 1789-1791 (1995).

2. P. Peumans et al., Nature 425, 158-162 (2003).

3. J. O. Ossó et al., Adv. Func. Mater. 12, 455-460 (2002).

4. N. Mott and R. Gurney, Electronic Processes in Ionic Crystals (Clarendon Press, Oxford, 1940).

5. P. Murgatroyd, J. Phys. D: Appl. Phys. 3, 151-156 (1970).

6. B. P. Rand et al., J. Appl. Phys. 98, 124902 (2005).

7. M. Bronner et al., phys. stat. sol. (a) 205, 549-563 (2008).

8. O. Berger et al., J. Mater. Sci.- Mater. El. 11, 331-346 (2000).

9. Z. Bao et al., J. Am. Chem. Soc. 120, 207-208 (1998).

10. D. G. de Oteyza et al., J. Am. Chem. Soc. 128, 15052-15053 (2006).

11. I. Salzmann et al., J. Appl. Phys. 104, 114518 (2008).

12. D. Datta et al., Thin solid films 516, 7237-7240 (2008).

13. T. Basova et al., phys. stat. sol. (b) 242, 822-827 (2005).

14. A. Opitz et al., SPIE Proc. 7002, 70020J (2008).

15. M. Pope and C. E. Swenberg, Electronic processes in organic crystals and polymers (Oxford University Press, New York, 1999)

16. M. Knupfer and H. Peisert, phys. stat. sol. (a) 201, 1055-1074 (2004). 